\title{
EMBRAER: de campeón nacional a jugador global
}

\section{Andrea Goldstein \\ Centro de Desarrollo, Organización de Cooperación y Desarrollo Económicos (OCDE) Andrea.goldstein@oecd.org}

Se ha escrito mucho sobre los aspectos macroeconómicos de la reforma económica en América Latina, pero mucho menos sobre sus resultados microeconómicos, en especial a nivel de la empresa. Este artículo examina cómo la empresa brasileña de aeronáutica, la EMBRAER, tras su privatización ha llegado a ocupar un lugar de vanguardia en el mercado mundial de esta industria de alta tecnología, tradicionalmente dominado por empresas con sede en países de la Organización de Cooperación y Desarrollo Económicos (OCDE). Concluye que los gerentes han desempeñado un papel decisivo en adaptar las innovaciones a las capacidades dinámicas de la empresa; que ésta ha logrado aliarse exitosamente con socios extranjeros y fortalecer su posición negociadora y que, tras ceder su control directo, las autoridades brasileñas la han apoyado sostenidamente. Sin embargo, para hacer extensivos estos resultados a otras empresas del país hay que compatibilizar los instrumentos para fomentar las exportaciones con las normas multilaterales. 


\author{
'Nos interesa lo que se refiere a la ingeniería ... \\ no somos fanáticos del aspecto comercial', \\ JuARez Wanderley, Presidente de la EMBraer \\ Financial Times, 6 de junio de 1995 \\ 'LOs aviones de la EMBRAER se fabrican \\ teniendo presente la satisfacción del cliente’ \\ Mauricio Botelho, Presidente de la EMBraer \\ InfoBrazil, 26 de mayo de 2000
}

\section{I}

\section{Introducción}

Históricamente, las grandes empresas industriales han cumplido una función decisiva en el desarrollo de tecnologías nuevas, la comercialización de nuevos productos y la introducción de nuevas formas de organización (Chandler, Amatori e Hikino, 1997). Así, han contribuido al crecimiento económico en Europa, América del Norte y Asia oriental, aunque obviamente pueden haber generado ineficiencias, en especial debido a su capacidad de cabildear en favor de políticas erradas (Goldberg y Maggi, 1999). Lo notable de América Latina es que la región es incapaz de crear empresas grandes, en especial de propiedad privada y/o orientadas a las exportaciones. Como es natural, en el sector privado hay no pocos grupos diversificados de empresas que son relativamente grandes - la verdad es que en la mayoría de los países en desarrollo constituyen la columna vertebral del mundo de los negocios- pero sirven principalmente al mercado interno y distan mucho de liderar el mercado mundial en sus respectivos rubros de actividad. Y hay también algunos grandes exportadores que suelen tener una participación importante en el mercado mundial de sus productos, pero son sobre todo entidades estatales que explotan recursos minerales no renovables, como petróleo y cobre.

Una de las pocas empresas manufactureras latinoamericanas que pueden arrogarse el título de líder

$\square$ Agradezco a Roberto Carlos Bernardes, Takahiro Fujimoto y a varios ejecutivos de EMBRAER nuestras conversaciones sobre el tema de este artículo, y a Edmund Amman, Steve McGuire, Richard Moxon, Nelson Silva y Paulo Bastos Tigre, así como a los participantes en seminarios del Center for Brazilian Studies (Oxford), el INSEAD y la Universidad Nacional de Singapur, y a tres jueces anónimos, por sus observaciones y sugerencias. Se aplican los descargos de responsabilidad ordinarios; en especial, las opiniones expresadas en este artículo no reflejan necesariamente la posición de la oCDE, del Centro de Desarrollo de la OCDE, ni la de sus Estados miembros. mundial de su mercado es la brasileña EMBRAER, que compite con la Bombardier por el tercer lugar en la clasificación mundial de fabricantes de aviones y es más rentable que su archirrival canadiense. En esta industria, el éxito depende de la solidez del diseño y de la manufactura, ${ }^{1}$ del precio y los costos de operación de la aeronave y de los servicios de posventa que se proporcionen a los clientes; éstos son relativamente pocos pero se encuentran repartidos por el mundo. Los gastos de lanzamiento y de investigación y desarrollo, así como los riesgos en materia de supervivencia, son elevados, pero en el tiempo la reducción de costos es inusualmente grande debido al proceso de aprendizaje dinámico. Se trata en suma de un sector oligopólico, en el cual no sorprende comprobar que casi todos los países que actualmente venden aviones de categoría internacional ya se habían incorporado a la industria de la aviación a fines de la Primera Guerra Mundial.

El presente trabajo tiene un doble objetivo. Primero, enriquecer la bibliografía sobre los resultados de la privatización, examinando la evolución de la EMBRAER para establecer hasta qué punto los recursos, las competencias y la capacidad de aprendizaje - fundamentales para poder participar en los mercados mundiales en los años noventa - se acumularon en la época en que la empresa era estatal y de qué manera el traspaso de la propiedad permitió que se convirtieran en fuente de ventajas competitivas. Segundo, apor-

\footnotetext{
${ }^{1}$ La fabricación misma de aviones consta de tres etapas principales: i) fabricación de las piezas fundamentales (láminas y placas metálicas, piezas que se producen utilizando máquinas computarizadas y no computarizadas, y piezas prefabricadas); ii) montaje del componente principal, y iii) instalación final en la estructura de los distintos sistemas operativos de la aeronave (tales como tendido eléctrico y aparatos electrónicos).
} 
tar a lo ya escrito sobre las formas de intervención estatal que contribuyen a la transformación industrial, examinando el caso concreto de una industria de alta tecnología como es EMBRAER, para saber: de qué manera ella se relaciona con el aparato gubernamental brasileño y con la economía mundial, si una empresa latinoamericana exitosa de alguna manera difiere de sus competidoras de países más industrializados y en qué medida su dinámica se puede aplicar con éxito en otras industrias o países.

Tras haber señalado de qué trata este trabajo, es igualmente importante indicar lo que no trata. Ante todo, no pone a prueba cuantitativamente si la intervención estatal fue o no exitosa internamente (esto es, si los beneficios fueron superiores a los costos) e internacionalmente (es decir, si la intervención sirvió para aumentar la competencia en el mercado mundial de aviones a reacción para el transporte regional). ${ }^{2} \mathrm{Se}$ gundo, y relacionado con lo anterior, no pretende explicar las circunstancias en que los gobiernos prestan apoyo a una industria nacional exponiéndose a represalias externas. La literatura sobre política comercial estratégica hace ambas cosas, utilizando dos enfoques diferentes. Por lo que se refiere a modelos, convendría actualizar los resultados obtenidos por Baldwin y Flam (1989), que incluyeron a la EMBRAER en un estudio de tres países sobre el mercado de aviones de 30 a 40 plazas (commuter aircraft); en lo que hace a la ciencia política, Goldstein y McGuire (2002) estudiaron a la EMBRAER conjuntamente con su competidora canadiense, utilizando el marco desarrollado en Busch (1999).

En las secciones siguientes se reseña la evolución de la empresa, primero como empresa pública (sección II) y luego como empresa privatizada (sección III), con miras a identificar los focos de capacidad empresarial que se observan en el desarrollo ascendente de la EMBRAER en los años setenta y ochenta, las razones por las cuales posteriormente se produjeron dificultades y los elementos de continuidad y cambio que se han dado desde 1994. Enseguida se hace un análisis de estos factores (sección IV), mientras que las conclusiones (sección V) vinculan este estudio monográfico con la discusión más general sobre los resultados de la privatización, el papel del Estado en la transformación industrial y la contribución de las grandes empresas industriales a este proceso.

\section{II}

\section{La EMBRAER como empresa pública: 1969-1994}

\section{Los orígenes}

Las Fuerzas Armadas brasileñas se han venido preocupando activa y permanentemente de la industrialización desde el siglo diecinueve. En el decenio de 1940 fue surgiendo en ellas una orientación industrialistatecnocrática (Sikkink, 1991). Influyentes pensadores militares, como el general Carlos de Meira Mattos, concibieron una teoría geoestratégica sobre el lugar de

\footnotetext{
2 En 1991, la Unión Europea impidió que el consorcio franco-italiano ATR asumiera el control de De Havilland, basándose en que la empresa que resultaría de la fusión habría dominado el mercado de aviones de turbohélice. Con el tiempo, Bombardier compró a su rival canadiense más pequeño. $\mathrm{Si}$ se analizara detenidamente el mercado de aviones a reacción que se destinan al servicio regional, al que no se ha incorporado ATR, se podría determinar si, en la medida en que impidieron el surgimiento del monopolio de Bombardier, los subsidios a las exportaciones otorgados por Brasil han aumentado el bienestar de los consumidores.
}

Brasil en el sistema mundial, que se basaba en el éxito con que el país había sabido lograr la autonomía tecnológica e industrial. Por lo que toca al sector aeronáutico, las estrategias comprendían una política de reserva de mercado, financiamiento estatal y apoyo tecnológico al sector privado a través del Centro Tecnológico Aeroespacial (CTA), ${ }^{3}$ creado en 1945 en São José dos Campos, Estado de São Paulo, donde se disponía de energía eléctrica, un clima favorable y una excelente topografía (Campolina Diniz y Razavi, 1999). El CTA, organización rectora destinada a la investigación aeronáutica según el modelo del Massachusetts Institute of Technology, llegó a ser 'quizá la más avanzada institución de investigación de los países en vías de industrialización' (Dahlman y Frischtak, 1993, p. 437) y condujo a la creación de

\footnotetext{
${ }^{3}$ En 1971, el cTA pasó a denominarse Centro Técnico Aeroespacial.
} 
instituciones hermanas dedicadas a la enseñanza técnica (Instituto Tecnológico de Aeronáutica, ITA) y a las investigaciones aeroespaciales (Instituto de Pesquisas Espaciais, INPE).

Se construyeron diversos prototipos y algunos de ellos, como el Paulistinha, comenzaron a fabricarse. A principios del decenio de 1960 la economía brasileña perdió impulso y en 1964 el deterioro de la estabilidad política desembocó en un golpe de Estado y en 21 años de gobierno militar. Pese a que estaban plenamente a favor de la propiedad privada, los militares orientaron más sus esfuerzos a la planificación del desarrollo e incrementaron los recursos destinados a ciencia y tecnología. Según se dijo, por razones de seguridad nacional, Brasil no podía darse el lujo de depender de aviones y repuestos importados, ni podía permitir que la fabricación de este material estratégico en el país fuese controlada por empresas extranjeras. La reactivación de la alianza entre los técnicos del sector público y los militares, que había sido tan importante para la creación de las empresas estatales en los decenios de 1940 y 1950 (Trebat, 1983), llevó a crear la Empresa Brasileña de Aeronáutica (EMBRAER) en 1969. ${ }^{4}$ Esta pertenecía mayoritariamente al Estado y heredó del CTA actividades orientadas a llevar adelante algunas misiones, en especial el proyecto IPD-6504 para la fabricación de un bimotor de turbohélice. Pese a que la relación entre la empresa y el Ministerio de Aeronáutica siguió siendo muy estrecha, se evitaron muchos trámites burocráticos y surgió un claro sentido de cometido empresarial. ${ }^{5}$ El ministerio no sólo manejó el mercado interno de manera favorable a EMBRAER, ${ }^{6}$ sino que concentró en sus propias manos la mayor parte de

\footnotetext{
4 Otras industrias importantes relacionadas con la defensa que se establecieron en São José dos Campos y que también surgieron del CTA son AVIBRAS (creada en 1961) para la fabricación de misiles y ENGESA (establecida en 1975) para la construcción de tanques y vehículos blindados. Se estima que en la década de 1980 AVIBRAS vendió 66 lanzacohetes Astros II a Iraq y un número no determinado a Arabia Saudita, Bahrein y Qatar. Entre 1982 y 1987 el total de ventas de Astros II ascendió a mil millones de dólares. Mientras que AVIBRAs ha dependido mucho de componentes importados, ENGESA desarrolló su propio diseño y capacidad técnica y se integró mejor con la base industrial local, en especial los fabricantes de piezas automotrices. Véase Brazil Special Weapons Guide en http:/ /www.fas.org/nuke/guide/brazil/indez.html.

${ }^{5}$ Entre 1969 y 1986 la composición del directorio siguió siendo la misma (Silva, 1998, p. 567).

${ }^{6}$ El gobierno y las Fuerzas Armadas de Brasil compraron aproximadamente un tercio de los Bandeirantes fabricados antes de 1980 . Por lo general, pagaron al contado y contribuyeron directamente a los gastos de inversión.
}

las responsabilidades financieras, fiscales, de comercialización y reguladoras y traspasó a la EMBRAER los compromisos internacionales. Además, la empresa pudo proporcionar opciones de financiamiento a sus clientes a través del Banco de Desarrollo Económico y Social (BNDES), un ente estatal; asimismo, aprovechó los beneficios que proporcionaba el Fondo de Financiamiento de la Exportación (FINEX), mecanismo de apoyo a las exportaciones administrado por el Banco do Brasil, y disfrutó de generosas franquicias tributarias. ${ }^{7}$ Finalmente, en 1973 el Ministerio de Relaciones Exteriores creó el programa especial PNEMEM (Política Nacional de Exportación de Material de Empleo Militar) cuya finalidad era promover las exportaciones de armamento.

\section{El ascenso}

La producción se inició en el decenio de 1970, en cooperación con asociados extranjeros, mediante la negociación de acuerdos de coproducción y de concesión de licencias que tenían por objeto poder ingresar rápidamente a los mercados sin demasiada dependencia tecnológica. Desde el primer momento se reconoció que era indispensable limitar el grado de integración vertical, para evitar el riesgo de una fragmentación excesiva de las operaciones comerciales, que en Brasil había hecho fracasar intentos anteriores de fabricar aviones (Silva, 1998, p. 177). ${ }^{8}$ En general, la EMBRAER se abstuvo de fabricar piezas caras y de alta tecnología y centró sus esfuerzos en el diseño de aeronaves, la fabricación de fuselajes y el montaje del producto final: ya en los años setenta suscribió acuerdos de compra a largo plazo con sus principales proveedores. Los dos modelos más populares —el biplaza de turbohélice para instrucción Tucano y el bimotor de turbohélice no

\footnotetext{
${ }^{7}$ Todas las empresas productoras de armamento estaban exentas del pago de derechos de importación por los insumos comprados en el exterior. Además, la EMBRAER estaba exenta de los impuestos al comercio (ICM) y a la producción (IPI). Por otra parte, las empresas brasileñas que compraran acciones sin derecho a voto de la EMBRAER podían obtener una rebaja de $1 \%$ del impuesto sobre los ingresos de sociedades. Asimismo, los organismos federales estaban obligados a comprar aviones de fabricación nacional cuando su valor no era más de $15 \%$ superior al de los productos competidores importados. Por último, en el caso de que hubiera productos brasileños similares disponibles, los aviones importados debían pagar un arancel de 50\%.

${ }^{8}$ Sin embargo, como la contratación de personal calificado de CTA/ ITA redujo el número de técnicos de estas instituciones, la EMBRAER terminó internalizando demasiado las actividades de investigación y desarrollo (Dagnino, 1993, p. 15).
} 
presurizado Bandeirante- eran de diseño nacional, aunque más de la mitad del valor de este último correspondía a piezas importadas. Una colaboración particularmente provechosa con socios italianos permitió producir el Xavante, avión a reacción para instrucción y ataque aéreo (con licencia de Aermacchi) y, mediante una empresa mixta con Aeritalia y Aermacchi, el AMX, un avión subsónico (Mach 0.86) de ataque de superficie para intercepción en el campo de batalla, apoyo aéreo a corta distancia y misiones de reconocimiento. Además, la EMBRAER amenazó con aumentar fuertemente los derechos de internación a fin de forzar a los productores extranjeros de aviones de uso general a aceptar un convenio por el cual se obligaban a proporcionar los equipos para montar el producto final en Brasil. Piper, que aceptó esta oferta, aumentó de manera espectacular su participación en el mercado a expensas de sus competidores, Beech y Cessna. Además de capacidad técnica, todos estos socios proporcionaron a la EMBRAER las técnicas de organización necesarias para la producción en serie. ${ }^{9}$ Dentro del Grupo Permanente de Movilización Industrial, en el que participaban desde 1965 las Fuerzas Armadas y la Federación de Industrias de São Paulo, la EMBRAER estableció una estrecha colaboración con empresas del sector privado brasileño, que proporcionaron una fracción cada vez mayor de los componentes finales. Además, la mitad de los miembros del directorio eran ejecutivos del sector privado (Silva, 1998, p. 256). Así pues, aunque los vínculos del capital eran relativamente débiles, puede estimarse que la industria aeronáutica constituye un buen ejemplo de la "triple alianza" entre empresas trasnacionales, empresarios privados nacionales y entidades estatales, que hasta comienzos de los años ochenta caracterizó la acumulación acelerada de capital que se produjo en Brasil (Evans, 1979).

Otra prioridad que se estableció desde el primer momento y que resultó decisiva para contrarrestar los gastos de desarrollo, fue un marcado acento en el mercado exportador. Esto permitió prolongar los ciclos de producción, alentó a los clientes a aportar ideas para la innovación tecnológica e impuso estrictas normas de desempeño. Para obtener la certificación en los prin-

\footnotetext{
9 A partir de los años ochenta, la EMBRAER ha sido el único subcontratista que diseña y produce alerones fuera de borda para el McDonnell Douglas MD-11, además del timón dorsal y los bordes marginales para el Boeing 777. También produce piezas fabricadas con máquinas de precisión para el Boeing 747 y el 767.
}

cipales mercados, los productos fabricados por la EMBRAER incluían sistemas de respaldo de las funciones principales, y los elementos estructurales de sus productos contemplaban márgenes de seguridad adecuados. Sin embargo, la empresa percibió acertadamente que tenía posibilidades de ocupar un lugar expectable en el mercado fabricando aviones que pudiesen operar en las condiciones más difíciles (climas extremos, pistas de aterrizaje no acondicionadas o sin pavimentar, apoyo terrestre mínimo) imperantes en regiones y países atrasados y cuyo mantenimiento fuese más fácil y económico. ${ }^{10} \mathrm{El}$ Bandeirante se sumó a la flota de varias aerolíneas regionales (commuter airlines) en los Estados Unidos, y en 1982 representó la tercera parte del mercado de esta clase de aviones para 10 a 20 pasajeros, desplazando a fabricantes estadounidenses (Dagnino, 1993, p. 9). ${ }^{11}$ Debido a que no contaban con el financiamiento ni con la capacidad para concebir un producto competitivo, rivales como Beech y Fairchild no pudieron reaccionar ante la llegada del Bandeirante. El mismo criterio caracterizó la producción de aviones militares provistos de menos adelantos técnicos que los que exportaban países industriales avanzados. El Tucano, que unía el bajo costo a soluciones técnicas innovadoras (por ejemplo, asientos eyectables y alineación en tándem de los asientos del alumno y el instructor), fue vendido a las fuerzas aéreas de Gran Bretaña y de Francia y se produjo en Egipto bajo licencia. El efecto neto en el acervo de divisas, sin embargo, fue poco importante y los efectos indirectos, de haberlos, nunca alcanzaron niveles críticos, entre otras razones por los obstáculos a la movilidad de la mano de obra que planteaba la doble tecnología (civil y militar) desarrollada y utilizada por los técnicos de la EMBRAER (Frischtak, 1992).

\section{La crisis}

La crisis de la deuda puso fin al período de financiamiento fácil que había favorecido el desarrollo de

\footnotetext{
${ }^{10}$ Los aviones con motores de turboventilación son menos poderosos que los a reacción, tienen alas más anchas y en consecuencia requieren pistas de menor longitud. Además, consumen menos combustible.

11 Como en el mercado de aviones el reconocimiento de marca es un elemento importante, al comienzo la EMBRAER tuvo grandes dificultades debido a que los usuarios no conocían el nombre y, además, a que los aviones fabricados en Brasil inspiraban muy poca confianza (Silva, 1998, p. 375).
} 
la industria aeroespacial brasileña, pero sólo vino a afectar a la EMBRAER a comienzos de los años noventa. Es cierto que a medida que la crisis económica de 1982 se dejó sentir en las empresas brasileñas, reduciendo drásticamente sus utilidades y en consecuencia el monto de los impuestos que pagaban, el mecanismo destinado a proporcionar capital privado para el financiamiento de la EMBRAER se agotó y la participación del Estado aumentó en la misma proporción. Sin embargo, dentro de las singulares características de la transición brasileña a la democracia, con posterioridad a 1985 los altos mandos de la Fuerza Aérea lograron oponerse a que se pusiera fin a su condición de ministerio y lograron que las partidas del presupuesto que le eran asignadas quedaran a cubierto de los llamamientos a la disciplina fiscal emanados del Ministerio de Hacienda y de las instituciones de Bretton Woods (Solingen, 1998). ${ }^{12}$

Se suponía que el EMB-120 Brasilia, el avión turbo presurizado para 30 pasajeros, derivado del Bandeirante y dado a conocer en 1985, se convertiría en el principal producto de la empresa. ${ }^{13}$ Para diseñar y fabricar esta aeronave se realizaron importantes inversiones en materia de uniones metal-metal, fresado químico y fabricación de materiales compuestos (Frischtak, 1992). Gracias a su bajo costo de operación, gran rapidez de despacho y velocidad de crucero relativamente elevada, el Brasilia tuvo un buen comienzo y captó un tercio del mercado total de aviones con capacidad para 30 a 40 pasajeros (Baldwin y Flam, 1989, cuadro 1, p. 493). ${ }^{14}$ Sin embargo, la EMBRAER se vio afectada por la recesión mundial de fines de los ochenta y por la decisión del gobierno brasileño de suspender las operaciones del FINEX. Además, la decisión de desarrollar al mismo tiempo un derivado más pequeño, el CBA-123, en cooperación con la Fábrica Militar de Aviones (FAMA),

\footnotetext{
12 En 1998, el presidente Cardoso logró finalmente reemplazar los ministerios administrados por cada rama de las Fuerzas Armadas por un ministerio de defensa único, dirigido por un civil, reforma que en el resto de América del Sur se había producido unos diez años antes.

${ }^{13}$ El avión ejecutivo Xingu, primera aeronave con cabina presurizada producida por la EMBRAER, fue un fracaso comercial, pero permitió que la compañía adquiriese conocimientos que luego utilizó para producir el Brasilia.

${ }^{14}$ Las simulaciones indican que los subsidios a las exportaciones lograron que las utilidades se trasladaran a la EMBRAER (Baldwin y Flam, 1989). En 1982, tras sostener que los subsidios otorgados a la exportación del Bandeirante eran ilegales, Fairchild planteó un reclamo ante la International Trade Commission de los Estados Unidos, el que en definitiva fue desechado.
}

de Argentina, resultó un fracaso. No obstante que era técnicamente avanzado, el avión terminó siendo demasiado costoso, entre otras razones porque la FAMA carecía de la capacidad necesaria para cooperar con la EMBRAER. Móviles políticos, como los de fortalecer la cooperación bilateral y crear confianza mutua con Argentina, prevalecieron sobre las consideraciones comerciales y, tras la partida de su presidente, Nestor Silva, en 1986 para asumir la presidencia de Petrobras, la EMBRAER no pudo hacer frente a la situación. Esto afectó la gestión de los flujos de caja: en una industria en la que es indispensable contar con financiamiento a largo plazo para asegurar su viabilidad durante el prolongado tiempo de puesta en marcha, la empresa acumuló deudas de plazos peligrosamente cortos. Como dice Frischtak (1992, p. 21), las técnicas y recursos acumulados en el proceso de desarrollo del AMX y el Brasilia permitieron que los técnicos de la EMBRAER tuvieran la "oportunidad" de fabricar un avión sobrediseñado (en el sentido de que no respetaba las restricciones del mercado, esto es, el precio). Pese a que la EMBRAER logró hacerse competitiva y asegurar una presencia importante en los mercados de ultramar, no sorprende que las consideraciones técnicas hayan prevalecido sobre las de mercado, debido a que la empresa dependió por mucho tiempo de las compras del sector público. Los militares atribuyen gran importancia al rendimiento y, en rigor, fue en el contexto del proyecto AMX que la EMBRAER instaló un sistema CAD/CAM, de diseño y fabricación asistida por computadoras, y fue la primera empresa brasileña que dispuso de esa tecnología (Sbragia y Terra, 1993, p. 3).

Durante las presidencias de Collor y de Franco (1990-1994), la situación política obstaculizó aun más el ajuste económico, la privatización y las reformas estructurales. El término de la guerra entre Irán e Irak, ${ }^{15}$ la menor intensidad del conflicto de Angola y el mayor apoyo prestado por el gobierno de los Estados Unidos a los productores de su país, cerraron los mercados de exportación del equipo militar fabricado por la EMBRAER. A medida que aumentaron los atrasos y se

\footnotetext{
${ }^{15}$ Casi un $40 \%$ de las armas vendidas por Brasil entre 1985 y 1989 se envió a Irak. No se dispone de información financiera fidedigna acerca de la importancia que revestía para la EMBRAER el mercado del Medio Oriente. La compañía recibió pedidos del Tucano EMB312 (110 de Egipto en 1983, 80 de Irak en 1985, 50 de Irán en 1988 y 100 de Libia en 1986), del EMB-111 para patrullaje marítimo (dos de Argelia en 1982 y 8 de Libia en 1986) y del EMB-121 Xingu (25 de Libia en 1986).
} 
cancelaron los pedidos, se elevó el costo de gestión de las existencias de aviones no vendidos. Algunos de los proveedores especializados de la EMBRAER, tales como ENGESA y TECNASA, se declararon en quiebra y la tasa de desempleo de São José dos Campos duplicó la del Estado de São Paulo en su conjunto (Broinizi Pereira, 1991). ${ }^{16}$ Pese a los esfuerzos por diversificarse hacia los servicios y otras actividades, en 1994 las pérdidas de la EMBRAER ascendieron a 310 millones de dólares contra ventas por valor de 177 millones (cuadro 1) y cayó al $38^{\circ}$ lugar entre los grandes exportadores brasileños. El regreso de Silva en 1991 no contribuyó mucho a revertir esta baja, aunque puede haber sido decisivo para acelerar la ulterior privatización.

CUADRO 1

Brasil: Principales datos financieros

(En dólares de los Estados Unidos) ${ }^{a}$

\begin{tabular}{|c|c|c|c|c|c|c|c|c|c|c|c|}
\hline & $\begin{array}{l}\text { Promedio } \\
1985-1991\end{array}$ & 1992 & 1993 & 1994 & 1995 & 1996 & 1997 & 1998 & 1999 & 2000 & 2001 \\
\hline Entradas brutas & 440 & 333 & 261 & 177 & 295 & 390 & 772 & 1362 & 1861 & 2859 & 2929 \\
\hline Mercado interno (\%) & 45 & 68 & 62 & 60 & 61 & 65 & 16 & 11 & 5 & 2 & 2 \\
\hline Exportaciones $(\%)$ & 55 & 32 & 38 & 40 & 39 & 35 & 84 & 89 & 95 & 98 & 98 \\
\hline Civiles, incl. servicios & .. & .. & .. & .. & .. & .. & 88 & 84 & 94 & .. & 96 \\
\hline Defensa & .. & .. & .. & .. & .. & .. & 12 & 16 & 6 & .. & 4 \\
\hline Entradas netas & -62 & -258 & -116 & -310 & -253 & -122 & -31 & 114 & 227 & 353 & 467 \\
\hline Exportaciones & .. & .. & .. & .. & 193 & 264 & 634 & 1173 & 1692 & 2377 & 2617 \\
\hline Importaciones & .. & .. & .. & .. & 147 & 220 & 469 & 881 & 1178 & 1249 & 1723 \\
\hline Saldo neto & .. & .. & .. & .. & 46 & 44 & 165 & 292 & 514 & 1128 & 894 \\
\hline Inversiones & .. & .. & .. & .. & 105 & 102 & 81 & 122 & 147 & .. & .. \\
\hline Investigación y desarrollo & 117 & 24 & 35 & 55 & 53 & 75 & 72 & 146 & & .. & .. \\
\hline Número de empleados & .. & .. & .. & 6087 & 4319 & 3849 & 4494 & 6737 & 8302 & 10334 & 10900 \\
\hline $\begin{array}{l}\text { Ingresos por empleado } \\
\text { (miles de dólares) } \\
\text { Partidas pro memoria: } \\
\text { valores en millones } \\
\text { de dólares de } 1996\end{array}$ & .. & .. & .. & 29 & 68 & 101 & 172 & 242 & 247 & 302 & 269 \\
\hline Entradas brutas & .. & 363 & 278 & 184 & 301 & 390 & 757 & 1320 & 1778 & 2671 & 2678 \\
\hline Exportaciones & .. & .. & .. & .. & 197 & 264 & 622 & 1137 & 1617 & 2221 & 2393 \\
\hline Importaciones & .. & .. & .. & .. & 150 & 220 & 460 & 854 & 1126 & 1167 & 1575 \\
\hline Investigación y desarrollo & .. & 26 & 37 & 57 & 54 & 75 & 71 & 142 & .. & .. & .. \\
\hline
\end{tabular}

Fuente: EMBRAER y MDIC/SACEX (datos de la balanza comercial; las cifras para 2000 y 2001 corresponden al período enero-noviembre).

a Las cifras expresadas en dólares se obtuvieron utilizando el tipo de cambio promedio o el tipo de cambio final del dólar comercial en los períodos correspondientes, según si se refieren a la cuenta de ingresos o a los datos del balance, respectivamente.

${ }^{16}$ En enero de 1990, cuando AVIBRAS pidió la declaración de quie-
bra, se suspendieron todos los programas de la empresa. La nómina
había bajado de 6000 a 900 trabajadores y la compañía tenía cohe-
tes sin vender por valor de 90 millones de dólares. A comienzos de
los años noventa, la situación financiera de AVIBRAS mejoró y en
1994 celebró un acuerdo con la NASA para el desarrollo conjunto de un programa de fabricación de cohetes. ENGESA se ha fragmentado y algunas de sus empresas fueron vendidas a intereses privados, mientras que las compañías relacionadas con la fabricación de material de guerra fueron absorbidas por el Estado e integradas a IMBEL (Industria de Material Bélico). 


\section{III}

\section{La EMBRAER como empresa privada}

\section{La privatización}

Pese a la oposición de los militares y a la prolongada huelga de 1990, en enero de 1992 el deterioro de la situación económica llevó al gobierno a incluir a la EMBRAER en la lista de empresas públicas que se pondrían en venta. Tras seis intentos fallidos, en diciembre de 1994 un consorcio adquirió un interés mayoritario de $45 \%$ en 89 millones de dólares. El consorcio lo formaban inversionistas estadounidenses reunidos por Wasserstein Perella, compañía de inversiones de Nueva York; Bozano Simonsen, uno de los mayores conglomerados financieros del Brasil, y Previ y Sistel, o sea, los fondos de pensiones del Banco do Brasil y Telebras, respectivamente. ${ }^{17} \mathrm{El}$ gobierno aportó capital nuevo, se hizo cargo de la deuda (700 millones de dólares) y conservó el $6.8 \%$ del capital de la empresa, incluida una "acción preferente" que tenía poder de veto para, entre otras cosas, modificar el control y los fines de la empresa y concebir y modificar los programas de defensa (ya sea que el gobierno brasileño participara o no en esos programas). ${ }^{18}$ De acuerdo con ciertas cláusulas del convenio, la propiedad extranjera se limitó a $40 \%$ y se estableció una moratoria de seis meses sobre los despidos.

Los nuevos propietarios contrataron un ejecutivo externo para que dirigiera la empresa (pese a que hasta la fecha tres de los seis vicepresidentes ejecutivos son antiguos gerentes de la EMBRAER), han aportado 413.6 millones de reales desde 1996 para fortalecer el capital de la firma y han intensificado los esfuerzos por mejorar la composición y plazos de su cuantiosa deuda. Los métodos y procesos de producción fueron mejorados, incluso invirtiendo una elevada suma en

\footnotetext{
${ }^{17}$ En junio de 1995, cuando Wasserstein Perella no pagó la suma que se había comprometido a aportar, la compró Bozano Simonsen. En conjunto, los tres accionistas mayoritarios reúnen el $60 \%$ de las acciones ordinarias sin derecho a voto de la EMBRAER.

${ }^{18}$ El gobierno tiene derecho a nombrar a uno de los miembros del directorio y generalmente ha elegido a un general de brigada de la Fuerza Aérea. Hasta 2001, los accionistas mayoritarios eligieron el segundo miembro del directorio, también un general de brigada. En 2000, el entonces Ministro de Relaciones Exteriores Lampreia sostuvo que el representante nombrado por el gobierno podía ser un civil, tal vez un diplomático. Él mismo fue elegido director en enero de 2001, cuando se retiró del gobierno.
}

tecnologías de la información, lo que en julio de 1997 le valió la certificación Iso 9001 por calidad del diseño, producción, ventas y servicios posventa. Para ayudar a las líneas aéreas a arrendar aviones nuevos en vez de comprarlos, entregando aviones usados en parte de pago, se creó una financiera propia en las Islas Caimán. Para aumentar la flexibilidad, la interacción y la autonomía, se encargó a una consultora internacional que diseñara un nuevo organigrama que, en vez de basarse en especificaciones estrictas de las funciones, se estructurara en torno a proyectos.

Se contrataron externamente servicios como los de mantenimiento de las instalaciones, transporte, alimentación, seguridad y mantenimiento de la maquinaria. Tras el despido de 1200 empleados y 500 técnicos y obreros en junio de 1995 (Bernardes, 2000a, p. 292), a fines de 1996 la nómina quedó reducida a 3849 trabajadores. En mayo de 1996, los trabajadores aceptaron una rebaja de los salarios y la adopción de una semana laboral más breve pero flexible, a condición de que los sueldos de la administración también se rebajaran un $10 \%$. La productividad aumentó: entre 1990 y 1996 se redujo de 16 a 9 meses el tiempo necesario para fabricar un Brasilia. ${ }^{19} \mathrm{Se}$ crearon cuatro centros de apoyo en el extranjero y en junio de 1995, dando su primera señal de que había recuperado la confianza, la EMBRAER celebró un acuerdo de riesgo compartido con United TechnologiesSikorsky para desarrollar y fabricar el sistema de combustible, los estanques de combustible y el tren de aterrizaje del S-92, helicóptero bimotor civil accionado por turbinas, para 19 pasajeros. Este acuerdo, que según lo previsto generaría un $10 \%$ de los ingresos por concepto de ventas, permitió que la EMBRAER conociera importantes avances tecnológicos en el campo de los materiales nuevos (INVAR) y del diseño virtual (CATIA). ${ }^{20}$

\footnotetext{
${ }^{19}$ Business Week, 1996.

20 EMBraer utiliza CATIA (Computer-Aided Three Dimensional Interactive Application) como plataforma de modelación geométrica de tipo estándar para rediseñar los procesos de desarrollo de la fabricación de aviones y mejorar la integración de las actividades internas y las relaciones con los socios internacionales.
} 


\section{La recuperación}

En 1989, la EMBRAER resolvió aventurarse en lo que a la sazón era un mercado bien pequeño para los aviones a reacción que volaban itinerarios regionales - generalmente inferiores a mil kilómetros y con tiempo de vuelo de alrededor de una hora- con una aeronave de cincuenta plazas. El avance del proyecto ERJ- $145^{21}$ quedó interrumpido mientras la empresa trataba de encontrar un nuevo dueño, pero adquirió impulso tras la privatización porque los nuevos administradores estimaron que de su éxito dependía la propia supervivencia de la empresa. No puede decirse con certeza si el salto a la fabricación de aviones a reacción obedeció a razones de prestigio - el deseo de los técnicos de demostrar que eran capaces de fabricar un avión más avanzado - o a que se prestó cuidadosa atención a las señales que enviaba el mercado. La función de información comercial (inteligencia) sólo se creó oficialmente en la empresa en 1991. Lo que sí puede afirmarse es que el esfuerzo de desarrollo se produjo cuando las empresas de aviación regional empezaron a reemplazar los aviones de turbohélice en sus flotas con aviones a reacción. A medida que la liberalización de la industria llevó a los grandes transportistas a depender cada vez más del tráfico generado fuera de los grandes centros de actividad, las líneas aéreas que prestaban servicios en rutas secundarias se vieron obligadas a ampliar la extensión de sus itinerarios (el número de ciudades que atendían cada centro de actividad) y a aumentar la frecuencia de los servicios. Además, los pasajeros comenzaron a manifestar una abierta preferencia por los aviones a reacción, que son más rápidos, más versátiles y producen menos ruido y vibraciones que los turbopropulsados. Así, entre 1998 y 1999 el mercado de aviones a reacción aumentó más de $50 \%$, estimándose que en el próximo decenio llegará al menos a 56 mil millones de dólares (Financial Times, 2000). Las perspectivas ya favorables se acentúan aun más si se considera que en los Estados Unidos están próximos a vencer los acuerdos suscritos en 1980 entre las líneas aéreas y los sindicatos. Estos especifican que los transportistas que prestan servicios en rutas secundarias no podrán utilizar aviones de más de 70 pasajeros, disposición que se debe a que los pilotos de las grandes líneas temieron que si se les permitía hacerlo, el empleo se desplazaría hacia estas afiliadas de menor costo.

\footnotetext{
${ }^{21}$ A fines de 1977 se agregó el sufijo ERJ a los aviones de la EMBRAER, originalmente conocidos como EMB.
}

El ERJ-145 es más liviano, más económico y su costo de operación es $15 \%$ inferior al de su principal competidor, el CRJ-200 de Bombardier (cuya autonomía de vuelo y velocidad son levemente superiores y que se había puesto en circulación algunos años antes). ${ }^{22} \mathrm{Su}$ rendimiento técnico superior obedece en parte a la incorporación de la técnica de turboventilación desarrollada para el programa AMX, mientras que su precio competitivo se explica en cierta medida porque comparte un $30 \%$ de sus elementos con el Brasilia (incluidos el morro y la cabina) y por el menor costo de la mano de obra. El nuevo avión se exhibió en la feria de Farnborough en 1996 y obtuvo su primer contrato con Continental Express, filial de Continental Airlines. ${ }^{23}$ Tres años y ocho meses después, en septiembre de 2000, se entregó la unidad número trescientos del ERJ-145 a la British Regional Airlines. A manera de comparación, cabe señalar que Bombardier tardó casi siete años en entregar el mismo número de CRJ-200. En 1998, la empresa produjo el primer prototipo del ERJ-135, versión para 37 pasajeros del ERJ-145, con el que comparte $96 \%$ de sus elementos, lo que reduce el costo de construcción y torna más atractivo el avión más pequeño para los transportistas que ya utilizan el modelo más grande. ${ }^{24}$ Gracias a esta tendencia, en 1998 la EMBRAER volvió a ser rentable tras 11 años consecutivos de trabajar a pérdida (cuadro 1$).^{25}$ El empleo ha aumentado a más de 10000 personas y, como los trabajadores nuevos son obviamente más jóvenes y además un creciente número de ellos son mujeres, la tasa de sindicalización se redujo apreciablemente: de 48 a 28\% entre 1997 y 2000 (EMBRAER, 2000a, p. 79).

En la actualidad, las exportaciones representan el 90\% de las ventas totales y, según sostiene EMBRAER, esta empresa es la que más unidades vende para el transporte regional en el mercado mundial. La devaluación del real brasileño en 1999 redujo los gastos por

\footnotetext{
${ }^{22}$ Información proporcionada personalmente por Nelson Silva, Director Gerente de EMBRAER Europa.

${ }^{23}$ El ERJ-145 está dotado de motores Rolls Royce y de instrumental de vuelo, como indicadores para los motores, sistemas de alerta a la tripulación y sistemas digitales de control de vuelo, producidos por Honeywell.

${ }^{24}$ Por ejemplo, para obtener su licencia para pilotar todos los aviones de una misma familia, los pilotos sólo necesitan recibir instrucción en cualquier avión de esa familia; además, se puede utilizar el mismo equipo de apoyo de tierra. En 1998 también se autorizó la fabricación de una versión de largo alcance del ERJ-145, que opera en rutas de hasta 3000 kilómetros y en julio de 2001 se entregó a American Eagle el ERJ-140, primer modelo del avión a reacción para 44 pasajeros.

${ }^{25}$ Los resultados operativos han sido favorables desde 1996.
} 
concepto de salarios de 13 a $9.7 \%$ de los costos de producción entre 1997 y 1999 (EMBRAER, 2000a, p. 43), pero también tornó más caro obtener créditos nuevos y cumplir con el servicio de la deuda pendiente en dólares, y redujo el valor en dólares de los recursos destinados a los programas de exportación del gobierno. Por esta razón, la empresa debió intervenir directamente para ayudar a algunos clientes a renegociar los arreglos financieros o, si ello no fue posible, otorgar reajustes de precios especiales. Además, la EMBRAER se ha convertido en el principal exportador de Brasil, y en 1999 generó un $3.5 \%$ del total de ventas externas del país. Aunque la empresa compra en el exterior aproximadamente la mitad de los insumos, ${ }^{26}$ con lo cual pasa a ser el segundo importador del país, en el período eneronoviembre de 2001 registró un saldo neto positivo de 984 millones de dólares. En 2001, EMBRAER obtuvo importantes utilidades comprando piezas al comienzo del año, cuando el real era fuerte, y vendiendo más adelante los aviones completos a un dólar por el que obtenía más reales.

\section{Los desafíos del futuro}

En la Feria del Aire de París, de 1999, la embraer anunció su intención de fabricar una familia de aviones de 70 a 108 asientos, contratando externamente la producción de alrededor de $80 \%$ de los componentes (pero no el motor ni el diseño de las alas), con lo cual prácticamente duplicaría su producción. Antes de comenzar a producir, la junta asesora de EMBRAER sostuvo una serie de reuniones con eventuales clientes, a fin de incorporar sus observaciones en el diseño del avión. ${ }^{27}$ El vuelo inaugural del ERJ-170 tuvo lugar el 19 de febrero de 2002 y las primeras entregas se harán a fines de 2002, mientras que la aeronave más grande estará disponible en 2004. La línea aérea suiza Crossair, que es la línea aérea regional más importante de Europa, será la primera en aprovechar las ventajas técnicas y comerciales (incluido el factor de pres-

\footnotetext{
${ }^{26}$ Folha de S. Paulo, 2000.

27 A raíz de esto, por ejemplo, la EMBRAER se convenció de que tenía que utilizar dos motores bajo las alas, en vez de los motores de cola como lo indicaba el diseño del ERJ-145 y del ERJ-135, lo que simplifica el servicio de la aeronave y da más comodidad a los pasajeros. Esta solución permite cargar el equipaje y abastecer los servicios de alimentación por el costado del avión que tiene el motor apagado. Mientras se da servicio al avión en tierra, el motor del otro lado puede seguir proporcionando energía eléctrica y aire acondicionado. Además, esta configuración debiera contribuir a que el operador reduzca al mínimo el tiempo de maniobras en tierra.
}

tigio) que lleva consigo la explotación de un modelo de avión enteramente nuevo. ${ }^{28}$

El rápido aumento de los pedidos sin despachar plantea importantes retos a la producción y cuando se inicie la fabricación de la nueva línea de aviones pueden surgir problemas de programación. Tan sólo para entregar los aviones comprometidos (es decir, aquéllos respecto de los cuales el cliente ha suscrito contratos acompañados del pago de una suma al contado), ${ }^{29}$ en 2000 EMBRAER tenía que aumentar en $33 \%$ su capacidad mensual de producción (EMBRAER, 2000a, p. 14). Además de aviones a reacción regionales, la EMBRAER participa en una serie de proyectos de defensa, aeroespaciales y de aviación general (cuadro 2). Se inició la construcción de una planta manufacturera y sitio de pruebas por valor de 150 millones de dólares en Gavião Peixoto (una localidad situada a unos 280 kilómetros al noroeste de São Paulo y libre de neblina), lo que va a permitir realizar en Brasil las pruebas de aeronavegabilidad de aviones militares de alta velocidad, en vez de tener que enviarlos a la base del lago Moses en los Estados Unidos.

En noviembre de 1999, Aerospatiale-Matra, Dassault Aviation y Thomson-CSF adquirieron cada una un interés de 5.5\% en la EMBRAER, y SNECMA un 3\% adicional, con lo cual el valor de la empresa se elevó a cerca de mil millones de dólares. Las acciones francesas no tendrán derecho a voto, pero percibirán un dividendo $10 \%$ mayor y permitirán que los nuevos socios nombren a dos representantes en el directorio de 11 miembros. La propuesta del consorcio francés superó las ofertas competidoras de BA у LockheedMartin, ${ }^{30}$ ambas deseosas de realizar un negocio que mejorara sus posibilidades de adjudicarse un pedido posiblemente importante para el programa de modernización FX-BR de la Fuerza Aérea brasileña, que supuestamente exigirá que se reconozca a la EMBRAER una

\footnotetext{
${ }^{28}$ La Crossair prefirió a la EMBRAER sobre las propuestas competidoras de Fairchild-Dornier y Bombardier. De acuerdo con un informe preparado por funcionarios de la oficina comercial de los Estados Unidos con base en Zurich, los principales factores que inclinaron la balanza en favor del fabricante brasileño fueron el peso total, el diseño de la cabina (un solo pasillo con dos asientos por lado, en vez de uno solo con filas de dos y tres asientos, como los aviones a reacción de la Fairchild), cierto nivel de aceptación del producto y una política de precios agresiva (Galambos, 2000).

${ }^{29}$ En la venta de aeronaves para transporte regional, la EMBRAER acostumbra exigir una cuota al contado en el momento de la firma del contrato de compraventa y tres cuotas parciales de $5 \%$ del valor de la venta a 18, 12 y seis meses antes de la entrega programada. Por lo general la cuota de pie y los pagos parciales no son reembolsables.
} ${ }^{30}$ The Daily Telegraph, 1999. 
CUADRO 2

\section{Brasil: Cartera de proyectos de la EMBRAER (sin contar los aviones a reacción para servicio regional)}

\begin{tabular}{|c|c|}
\hline Nombre & Descripción y volúmenes de producción \\
\hline AL-X & $\begin{array}{l}\text { Fabricado con arreglo a un contrato suscrito con la Fuerza Aérea de Brasil (FAB). Desde } 1995 \text { han estado operando } \\
\text { dos prototipos. El vuelo inaugural del primer avión anterior a la puesta en producción tuvo lugar en mayo de } 1999 \\
\text { y la primera entrega se prevé para 2001. Estos aviones, cuya potencia es dos veces superior a la del Tucano estándar, } \\
\text { se utilizarán para instrucción avanzada de pilotos y para operaciones de defensa en el Amazonas. }\end{array}$ \\
\hline AMX & $\begin{array}{l}\text { El proyecto surgió de las especificaciones de } 1997 \text { para el cazabombardero táctico de tamaño pequeño de la } \\
\text { fuerza aérea italiana. En 1980, la EMBRAER se incorporó a la sociedad Aeritalia/Aermacchi original. El primer } \\
\text { prototipo se puso en vuelo en mayo de 1984, la producción se inició a mediados de } 1986 \text { y el primer avión de } \\
\text { la serie de } 30 \text { unidades ( } 21 \text { para Italia y } 9 \text { para Brasil) se terminó de fabricar en Turín en marzo de } 1988 \text {. En } 1988 \\
\text { se suscribió un nuevo contrato (por } 59 \text { unidades para Italia y } 25 \text { para Brasil) y al año siguiente se inició la } \\
\text { producción para la Fuerza Aérea brasileña. A comienzos de } 1992 \text { se autorizó la fabricación de una tercera serie } \\
\text { y en mayo de } 1992 \text { se entregó el primer biplaza para la Fuerza Aérea brasileña. }\end{array}$ \\
\hline
\end{tabular}

AMX-T Versión mejorada del AMX. La fabricación se inició en Italia a fines de 1994 y se interrumpió en 1998 (se produjeron 110 monoplazas y 26 biplazas) y continúa en Brasil. Actualmente operan alrededor de 190 unidades en las Fuerzas Aéreas de Italia y Brasil. En septiembre de 1999 el AMX-T se adjudicó una propuesta para Venezuela.

EMB-145 AEW\&C y RS En mayo de 1999 se dieron a conocer versiones especiales del ERJ-145. El moderno sistema de radar del AEW\&C, desarrollado por Ericsson, tiene capacidad para llevar a cabo misiones de supervisión, patrullaje y control de tráfico aéreo. El RS fue diseñado para misiones de telemedición de superficies, control ambiental y estudios geológicos. Se prevé que desempeñará un papel decisivo en el Amazon Surveillance System (SIVAM) que está desarrollando Raytheon a un costo de 1.2 mil millones de dólares. La Fuerza Aérea griega ha encargado a la EMBRAER cuatro AEW\&C, que utilizará en su espacio aéreo para el sistema de alerta temprana y control dentro del territorio de la OTAN. De acuerdo con el mismo contrato se adquirió un ERJ 135 para misiones especiales de transporte y apoyo. México también prefirió a la EMBRAER para la compra de un AEW\&C y dos RS, que destinará al patrullaje marítimo.

EMB-314 Super Tucano Monomotor de turbohélice para la instrucción de pilotos y misiones de aerorreconocimiento armado para la defensa. A la fecha se han producido unas 650 unidades de la primera versión del EMB-312 Tucano, las que prestan servicios en 15 Fuerzas Aéreas en el mundo. Actualmente se está desarrollando el modelo AL-X (avión ligero de ataque), provisto de un motor de 1600 SHP, aviónica moderna y otras innovaciones tecnológicas, en versiones monoplaza y biplaza. En agosto de 2001, la Fuerza Aérea Brasileña (FAB) firmó un contrato por 76 unidades a firme y otras 23 opcionales, para misiones básicas/tempranas de entrenamiento avanzado, incluida la instrucción para utilizar el armamento. La producción se inició en febrero de 2002.

Estación Espacial Internacional

Proyecto internacional en el que intervienen 13 países de la OCDE, Rusia y Brasil, para el montaje de una plataforma conectada al exterior de la Estación Espacial Internacional para la realización de experimentos. La Agencia Espacial Brasileira, que coordina el Programa Nacional de Actividades Espaciales (PNAE), seleccionó a la EMBRAER, que trabaja con la Boeing.

Derivaciones Versión modificada del ERJ-135 que se produce en modelos ejecutivo, para shuttle de empresas y VIP, dados a conocer en el festival aéreo de Farnborough en 2000. Agregándole dos estanques de combustible adicionales, la versión ejecutiva tiene una autonomía de vuelo de 3200 millas náuticas, esto es, suficiente para volar de costa a costa a través de los Estados Unidos o ir de París al Medio Oriente. El vuelo inaugural se efectuó en marzo de 2001 y obtuvo la certificación de Brasil. En marzo de 2002, la EMBRAER recibió 25 pedidos a firme y 50 opciones de compra.

Fuente: Elaboración del autor, basada en datos de EMBRAER y otras fuentes.

participación decisiva en el negocio. ${ }^{31}$ Se prevé que dentro de poco la EMBRAER y los industriales franceses promuevan conjuntamente el Mirage 2000 y el avión de combate Rafale, además de los turboventiladores y los programas electrónicos que producen Snecma

\footnotetext{
${ }^{31}$ Antes de 2005 se necesitarán de 75 a 150 aviones para reemplazar los envejecidos F-5 y Mirage, con un costo total de hasta 6 mil millones de dólares. En agosto de 1997, la administración Clinton anuló la prohibición por 20 años de la venta de armamento y aviones estadounidenses de alta tecnología a América Latina.
}

y Thomson-CSF. ${ }^{32}$ Esta alianza estratégica aumentará las ventas de aviones militares de la EMBRAER, que

\footnotetext{
${ }^{32}$ En abril de 2000 EMBraER y sus socios franceses crearon el Mirage 2000 BR Consortium para comercializar en América Latina una nueva versión del Mirage 2000-5 Mk 2 de Dassault Aviation. Todas las actividades del nuevo consorcio serán coordinadas por la EMBRAER, que actuará como cabeza. Las actividades de análisis, desarrollo y prueba del Mirage 2000 BR serán llevadas a cabo en forma conjunta, ya sea en las instalaciones de la EMBRAER en Brasil, o en Francia. El montaje final se hará en la nueva planta de la EMBRAER en Gavião Peixoto.
} 
actualmente representan un $7 \%$ de sus entradas totales, así como su penetración en el mercado chino. ${ }^{33} \mathrm{La}$ decisión fue impugnada por los altos mandos de la Fuerza Aérea, molestos porque el gobierno no les consultó la decisión de respaldar el negocio y preocupados por la posibilidad de que la asociación de EMBRAER con Dassault les obligue a comprar equipo francés.
Tras haberse formulado observaciones particularmente graves, el gobierno llamó a retiro al comandante en jefe. ${ }^{34} \mathrm{El}$ procurador general de Brasil dictaminó que la venta no constituye un cambio de control y que, en vista de que no afecta los intereses nacionales del país, el gobierno no debería utilizar su acción preferente para impedir el negocio.

\section{IV}

\section{Interpretando la evidencia}

A lo largo de sus 25 años de historia como entidad estatal, la EMBRAER se valió de convenios de otorgamiento de licencias y de cooperación para atraer a la empresa recursos y conocimientos nuevos y desarrollar un sistema básico de competencias técnicas para la fabricación de aviones. Sin embargo, aunque al comienzo este proceso de aprendizaje se acompañó de avances en otros frentes - como técnicas de organización y comercialización- hacia mediados de los años ochenta las principales autoridades de la empresa se dejaron dominar por criterios que no eran de orden técnico. Los vínculos entre las diversas partes de la empresa, y particularmente entre Brasil y las filiales extranjeras que supuestamente debían advertir con antelación las exigencias del cliente, se debilitaron. Escribiendo en 1992, cuando la EMBRAER hacía frente a la crisis más severa de su corta vida, Frischtak reconoció que la empresa era capaz de explotar un nicho especial en el mercado mundial, pero destacó que el principal obstáculo para mantener este éxito a largo plazo era la falta de recursos financieros y de soluciones técnicas centradas en el mercado. Asimismo, expresó que lo primero que había que hacer para recuperarse era celebrar acuerdos de cooperación en materia de desarrollo, producción y comercialización.

\section{Gestión}

El repunte que se produjo después de la privatización obedeció en gran parte a las medidas adoptadas por

\footnotetext{
${ }^{33}$ En septiembre de 2000, Sichuan Airline recibió el primero de los cuatro ERJ-145 que ha adquirido. Este modelo es el primer avión de la EMBRAER que opera en China y, al mismo tiempo, el primer avión a reacción que coloca la empresa en el mercado de Asia sudoriental. En 2002, la empresa inauguró bodegas en el aeropuerto internacional de Beijing.
}

los nuevos ejecutivos, que inyectaron una nueva estrategia comercial destinada a asegurar que las competencias básicas de la EMBRAER se alinearan con las señales del mercado. Gracias a diversas reformas organizativas se han introducido innovaciones y han mejorado los resultados. La jerarquía se simplificó, al reducirse de diez a cuatro el número de grados ejecutivos; además, se aplicó a todos los funcionarios un sistema de remuneraciones basado en el rendimiento (Ghemawat, Herrero y Monteiro, 2000). Y lo que es más importante, se incorporaron oficialmente a las actividades habituales de la empresa la planificación estratégica, la gestión de la calidad total, la información (inteligencia) de mercado, la estrategia kaizen de habilitación de la fuerza de trabajo y el intercambio de información sobre los resultados del sistema (Bernardes, 2000a).

El proceso de cambio organizacional involucra “visión”, aprendizaje mediante la experimentación y la retroalimentación, y construcción de consensos (Fujimoto, 2000). Aunque todos estos elementos han cumplido una función, no es fácil señalar cuánto ha pesado cada uno; es posible que el más importante haya sido el primero. En 2001, Mauricio Botelho, Gerente General de la EMBRAER desde 1994, obtuvo el premio Laurels que otorga la principal publicación de la industria 'por haber sabido interpretar acertadamente el desplazamiento de la industria del transporte aéreo regional desde aviones de turbohélice a aviones a reacción -percepción que escapó a muchos conocidos fabricantes europeos y estadounidenses- $\mathrm{y}$ haberse centrado en

\footnotetext{
${ }^{34}$ La controversia sobre la EMBRAER coincidió con el intento del gobierno de quitarle a la Fuerza Aérea la administración del sistema de aeropuertos del país, con miras a su posible privatización (véase Veja, 2000).
} 
un solo objetivo fundamental: satisfacer al cliente' (Aviation Week and Space Technology, 2001). Cabe recordar que en la EMBRAER la función de información comercial (inteligencia de mercado) se creó oficialmente después y no antes de que se lanzara la línea de aviones ERJ. Aunque Botelho era ingeniero mecánico, no sabía nada de actividades aeroespaciales, lo que posiblemente le haya hecho más fácil detectar la transformación de la industria.

El programa iniciado en 1997 de transformación de la organización para obtener resultados, conocido como Programa TOR (Transforming the Organization for Results), ha sido la base del proceso de aprendizaje. En una fase de crecimiento acelerado, ha procurado aunar el desarrollo de actividades nuevas con la revisión y mejora permanente de los procesos operativos. El TOR comprende iniciativas empresariales como las de desarrollo tecnológico integrado (en las esferas de la producción, las técnicas de producción y la tecnología de la información), proyectos de emergencia, depuración de datos y aplicación del sistema de planificación de los recursos de la empresa conocido como ERP (Enterprise Resource Planning). Por lo que toca al desarrollo personal y la capacitación de su personal, la EMBRAER ha introducido un plan de desarrollo profesional y salarios, un programa de integración industrial y un plan de jubilación complementario. En 2000, el valor de las prestaciones medicohospitalarias y sociales otorgadas a los trabajadores ascendió a 124 millones de reales, es decir, aumentó $81 \%$ en un año. Finalmente, en 2000 el programa de participación en las utilidades distribuyó 81 millones de reales, comparado con 36.6 millones en 1999 (EMBRAER, 2000b).

\section{Localización de la empresa}

São José dos Campos está situado en el corazón del valle del Paraíba, y lo constituyen 43 municipios que albergan a 430 exportadores y producen un $3 \%$ del producto interno bruto de Brasil. Empresas trasnacionales como Ericsson, Volkswagen, Ford y General Motors han levantado allí algunas de sus plantas más grandes del mundo y atraído inversiones adicionales a las industrias de componentes y electrónica. La EMBRAER pudo aprovechar estas inversiones, actuando como punto de apoyo y coordinadora de una red de proveedores especializados de piezas separadas, esto es, proveedores de nivel II (Bernardes, 2000b). Pese a que la EMBRAER no tiene intereses patrimonia- les en ellas, algunas fueron creadas a mediados de los años noventa por técnicos especializados que habían sido despedidos por EMBRAER y otras empresas aeroespaciales (cuadro 3). Actualmente se lleva a cabo un esfuerzo concertado por mejorar y profundizar la articulación entre los distintos actores de los sectores público y privado, bajo el liderazgo de la federación industrial de São Paulo (FIESP/CIESP), el Servicio de Apoyo a las Micro y Pequeñas Empresas (SME) del Servicio Brasileño de Apoyo a las EmpreSas (SEBRAE) y las asociaciones comerciales locales (la Asociación Comercial e Industrial de São José dos Campos y la Cámara de Comercio del Valle del Paraíba). La idea del programa Exporta Vale es convertir a la región en el polo de alta tecnología del Brasil, con fuerte presencia de actividades emprendidas a nivel local, y aumentar a mil el número de empresas exportadoras y a 500 millones de dólares el valor de las ventas externas en 2003 .

Las actividades de investigación y desarrollo —como el análisis mecánico, las pruebas térmicas, las pruebas de compatibilidad electromagnética y las simulaciones en el vacío- se han entregado al CTA. En el marco del programa Parceria para Inovação Tecnológica (PITE), financiado por la Fundação de Amparo a Pesquisa do Estado de São Paulo (FAPESP) y cuya finalidad es reforzar la colaboración entre los sectores privado y académico, la EMBRAER y el ITA también han emprendido una serie de proyectos (cuadro 4). Sin embargo, según Bernardes (2000b), las vinculaciones entre ésta y otras instituciones técnicas locales, como la Escuela de Ingeniería de São Carlos y la Escuela Politécnica, se han ido debilitando a medida que EMBRAER recurre cada vez más a organismos técnicos extranjeros ${ }^{35}$ y crea sus propias escuelas técnicas para capacitar a su personal. En marzo de 2001 se inició el programa de especialización técnica en el campo de la aeronáutica, iniciativa que tiene por objeto proporcionar capacitación técnica a profesionales recién titulados y a quienes tienen hasta dos años de experiencia profesional. El programa constituye el primer paso para la creación de la Universidad Empresarial EMBRAER.

\footnotetext{
35 Las pruebas transónicas en túnel aerodinámico se llevan a cabo en el laboratorio aeroespacial nacional NLR de los Países Bajos y las de baja velocidad en el Instituto aerohidrodinámico central de Rusia (TsAGI). El objetivo principal de estas pruebas es examinar el funcionamiento de las alas en diversos segmentos del plan de vuelo previsto.
} 
CUADRO 3

\section{Brasil: Algunas empresas aeroespaciales del valle del Paraíba}

\begin{tabular}{ll}
\hline Empresa & Descripción \\
\hline $\begin{array}{l}\text { AEROSERV Serviços } \\
\text { Aeronáuticos }\end{array}$ & $\begin{array}{l}\text { Creada en } 1999 \text { como microempresa dentro de la planta de la EMBRAER, ha evolucionado rápidamente hasta } \\
\text { incorporarse a la categoría de empresa mediana o pequeña y en 2001 tuvo ventas por } 5 \text { millones de dólares. En } \\
\text { la actualidad, tiene su propia planta en Jacareí y cuenta con 200 operarios, } 80 \% \text { de los cuales habían trabajado } \\
\text { en la EMBRAER. }\end{array}$
\end{tabular}

AKROS

Fundada a comienzos de los años noventa, se especializa en análisis estructural y en el diseño de piezas mecánicas, ingeniería de producto y procesos de fabricación. Además, ha participado activamente en el programa aeroespacial, en la especificación de pruebas dinámicas y estáticas, el análisis estructural, el diseño de herramientas mecánicas y la preparación y revisión de la documentación técnica.

CENIC

Diseña, produce y prueba materiales compuestos (fibra de carbón) para piezas y conjuntos de piezas mecánicos. Subcontrata la fabricación, entre otros, de tapas de motor compuestas y toberas para el VLS-1 (vehículo lanzacohetes).

COMPSIS

Creada en 1989, desarrolla y produce sistemas de transporte de control numérico y mecanismos de prueba para la industria automotriz. Las técnicas de desarrollo y producción que utiliza cumplen con las normas DOD-STD2167 y ANSI/IEEE exigidas para adquirir, desarrollar o prestar apoyo a sistemas computacionales fundamentales para el cumplimiento de misiones.

DIGICON

Inició sus actividades en el campo de los transductores y procesadores para máquinas de control numérico. También produce trazadores de gráficos y discos duros, así como algunos programas CAD. Fabrica componentes mecánicos para piezas estructurales de los satélites y subsistemas del Instituto Nacional de Pesquisas Especiais (INPE) y, en colaboración con la MBB de Alemania, se encarga del montaje de paneles satelitales solares.

ELEBRA

Produce, vende y distribuye equipo electrónico para comunicaciones y piezas electrónicas (radares, tableros de control del tráfico aéreo, antenas y transmisores de alta frecuencia) y presta servicios industriales y de ingeniería civil. De acuerdo con informaciones proporcionadas por la empresa, en 1999 sus ventas ascendieron a 15 millones de dólares. Fue adquirida por el grupo NORCAL. En el marco del programa CBERS (China-Brazil Earth Resource Satellite Cooperation) se encargó de desarrollar computadores para uso a bordo, sistemas de control de posición y órbita y el diseño de transmisores UHF.

Gespi Aeronáutica Establecida en 1974 para el diseño y la fabricación de piezas y componentes para EMBRAER. A partir de 1990 su división aeroespacial ha desarrollado otros productos, para apoyo en superficie, limpieza de aerocomponentes y sistemas de cohetes antineblina.

MECTRON

Fundada por egresados del ITA, fabrica sistemas para la defensa, tales como simuladores para misiles antitanques, equipos electrónicos de uso médico y sistemas de control de tráfico. En el campo de las actividades aeroespaciales, ha desarrollado sistemas eléctricos, componentes electrónicos y programas de control satelital, así como algunas plataformas para la recopilación de datos. En 2001, la Fuerza Aérea italiana adjudicó a Mectron y Alenia un contrato por 45 millones de dólares para la instalación del radar SCP-01 en su flota de AMX.

N\&N Informática e Automação

Qualitas Engenharia

TECNASA

Tectelcom Aerospacial
Desarrolla programas a pedido para el procesamiento de imágenes, procesos de control y mecanización de la línea de productos. Sus clientes incluyen, entre otros, a la Fuerza Aérea y la Armada de Brasil.

Establecida en 1993 para desarrollar sistemas de gestión. Entre sus clientes cabe mencionar a HELIBRAS y EMBRAER (desarrollo de aplicaciones para simular las relaciones pre y posventa con los clientes y para prestar apoyo a éstos).

Fundada por egresados del ITA, se especializa en la fabricación de equipo electrónico para el apoyo a la navegación aérea, como el radar multimodal de combate aéreo (para el AMX) y varios sistemas de contramedidas. La empresa fabricó transpondedores de telemetría y telecomando para los proyectos MEC-B y CBERS y en sus instalaciones de prueba de diseños ha definido las especificaciones de varios productos.

Filial del grupo Tectelcom.

Fuente: Elaboración del autor basada en información proporcionada por las empresas y otras fuentes.

\section{Los socios}

Varias firmas americanas y europeas proveedoras de piezas para las empresas aeroespaciales europeas y americanas entregaron su aporte a la fabricación del ERJ-145 en calidad de socios de riesgo compartido, invirtiendo directamente 64 millones de reales (de un total equivalente a 592.7 millones de reales, o aproximadamente 300 millones de dólares) en efectivo y materiales, y proporcionando liquidez pactando el pago diferido de las obligaciones. Los proveedores de nivel I (cuadro 5) entregan sistemas completos —en vez de 


\begin{tabular}{|c|c|c|c|c|}
\hline & Socio & $\begin{array}{l}\text { Presupuesto } \\
\text { total (dólares) }\end{array}$ & $\begin{array}{l}\text { Participación EMBRAER } \\
\text { (porcentaje) }\end{array}$ & Calendario \\
\hline $\begin{array}{l}\text { Desarrollo de un sistema DGPS para posicionamiento } \\
\text { de aeronaves en tiempo real }\end{array}$ & INPE & 218117 & 0 & $\begin{array}{l}\text { Marzo } 2002- \\
\text { agosto } 2003\end{array}$ \\
\hline $\begin{array}{l}\text { Aplicaciones avanzadas de la mecánica electrónica } \\
\text { de los fluidos a aviones de alto rendimiento }\end{array}$ & ITA & 1255000 & 8 & $\begin{array}{l}\text { Enero 2002- } \\
\text { diciembre } 2004\end{array}$ \\
\hline $\begin{array}{l}\text { Identificación de los derivados de la estabilidad y } \\
\text { el control aeroespacial }\end{array}$ & ITA & 195525 & 0 & $\begin{array}{l}\text { Enero 2002- } \\
\text { junio } 2003\end{array}$ \\
\hline $\begin{array}{l}\text { Desarrollo de la técnica de prueba aerodinámica } \\
\text { para aeronaves de alto rendimiento }\end{array}$ & ITA & 948479 & 0 & $\begin{array}{l}\text { Junio } 2001- \\
\text { mayo } 2004\end{array}$ \\
\hline
\end{tabular}

Fuente: Fundação de Amparo a Pesquisa do Estado de São Paulo (FAPESP).

piezas separadas - y asumen la gestión de la cadena de abastecimiento mediante la subcontratación de las piezas independientes a proveedores de nivel II. En el caso de la línea ERJ 170/190, los costos de desarrollo son aún mayores (cerca de 850 millones de dólares) y constituyen con mucho la mayor inversión que haya jamás emprendido la EMBRAER. No menos de un tercio de estos costos serán aportados en efectivo por los socios, quienes serán responsables de desarrollar, producir y entregar tanto sistemas completos como piezas sofisticadas. La selección de los principales socios de riesgo compartido y la negociación de contratos tardaron un año de inicio a término. Se enviaron las bases de las propuestas a 85 empresas, 58 de las cuales presentaron ofertas. La EMBRAER se encargará de producir el fuselaje delantero, la proa, la punta del fuselaje, la sección II del fuselaje y el carenado desde el plano de sustentación al fuselaje. Algunos proveedores extranjeros como Pilkington Aerospace, Parker Hanefin y SONACA ya han iniciado operaciones en Brasil, y otros como Latecoere tienen intenciones de hacerlo (Gazeta Mercantil, 2000). Además, junto con Liebherr, de Alemania, la EMBRAER creó ELEB, empresa mixta para producir trenes de aterrizaje y piezas hidráulicas, que en noviembre de 2000 se fusionó con Órbita Sistemas Aeroespaciais.

Esta estrategia no sólo debería reducir los gastos y riesgos de desarrollo de EMBRAER, sino que, al disminuir el número de proveedores y facilitar la logística, le permitiría centrar la atención en lo que sabe hacer mejor, esto es, concebir, montar y comercializar el producto final y brindarle servicio. ${ }^{36} \mathrm{El}$ calendario de

\footnotetext{
36 Véase la descripción del programa de desarrollo integrado de los productos de la EMBRAER en Araujo y Da Cruz (2000).
}

desarrollo tiene 38 meses de duración y también aspira a adelantar lo más posible la fase de resolución de problemas e identificar los problemas de interferencia, que son muy costosos en resolver si se identifican después del primer montaje completo (Thomke y Fujimoto, 2000). ${ }^{37}$ Técnicos de los socios de riesgo compartido son enviados a Brasil a trabajar con sus contrapartes brasileñas. En mayo de 2001 se inició la fase de definición conjunta del ERJ-190 con la Kawasaki Heavy Industries, que se encargará de la fabricación de las alas. Asimismo, se construyó un nuevo centro de realidad virtual que utiliza prototipos virtuales y visualización en gran escala para reducir el tiempo que tardan los aviones en llegar al mercado. Para producir maquetas digitales en lugar de modelos reales, la EMBRAER utilizará ENOVIAvpm (modelo de producto virtual) y CATIA, y se ha pedido a todos los proveedores de nivel I que los instalen para facilitar las comunicaciones. La maqueta electrónica original está centralizada en los computadores de la EMBRAER y para actualizar en línea el banco de datos se ha creado un sistema nuevo, exclusivo para este propósito, de intercambio electrónico de datos (EDI).

\section{Las políticas públicas}

Por último, instituciones del sector público como el BNDES y la Financiadora de Estudios y Proyectos (FINEP) perteneciente al Ministerio de Ciencia y Tecnología

\footnotetext{
${ }^{37}$ Por ejemplo, la utilización de la dinámica computarizada de líquidos, de maquetas digitales y de la realidad virtual permitió simular la introducción de alitas pequeñas en el ERJ-170. La EMBRAER resolvió instalarlas debido a que mejoran la combustión y la capacidad de elevarse del avión, además de su rendimiento en Hot \& High y su capacidad de franquear obstáculos.
} 
CUADRO 5

EMBRAER: Socios de riesgo compartido

\begin{tabular}{|c|c|c|}
\hline & ERJ $145-135$ & ERJ $170-190$ \\
\hline C/D (Estados Unidos) & $\begin{array}{l}\text { Interiores de la cabina y de las bodegas de } \\
\text { carga. }\end{array}$ & Interiores. \\
\hline ENAER (Chile, de propiedad estatal) & $\begin{array}{l}\text { Estabilizadores verticales y horizontales y } \\
\text { timones de profundidad. }\end{array}$ & \\
\hline $\begin{array}{l}\text { GAMESA (Grupo Auxiliar Metalúrgico) } \\
\text { (España) }\end{array}$ & $\begin{array}{l}\text { Alas, nacelas del motor y puertas principales } \\
\text { del tren de aterrizaje. }\end{array}$ & $\begin{array}{l}\text { Parte posterior del fuselaje y planos vertical } \\
\text { y horizontal de la cola. }\end{array}$ \\
\hline General Electric (Estados Unidos) & & $\begin{array}{l}\text { Turboventiladores, abastecedores de energía, } \\
\text { y nacelas del motor. }\end{array}$ \\
\hline Grimes Aerospace (Estados Unidos) & & Iluminación exterior y de la cabina del piloto. \\
\hline Hamilton Sundstrand (Estados Unidos) & & $\begin{array}{l}\text { Núcleo de cola, unidad auxiliar de energía, } \\
\text { sistemas eléctricos y sistema de ordenación } \\
\text { del aire. }\end{array}$ \\
\hline Honeywell (Estados Unidos) & & Equipos electrónicos. \\
\hline Kawasaki (Japón) & & $\begin{array}{l}\text { Talones de sustentación del ala, pilón del } \\
\text { motor, piezas fijas de aterrizaje y bordes de } \\
\text { salida, flaps, aerofrenos y superficies de con- } \\
\text { trol de vuelo de las alas. }\end{array}$ \\
\hline Latecoere (Francia) & & Partes del fuselaje central. \\
\hline Liebherr (Alemania) & & Trenes de aterrizaje. \\
\hline $\begin{array}{l}\text { SONACA (Societé Nationale de Construction } \\
\text { Aérospatiale) (Bélgica, de propiedad estatal) }\end{array}$ & $\begin{array}{l}\text { Partes del fuselaje central y posterior, repa- } \\
\text { raciones, puertas principales y del equipaje, } \\
\text { y góndolas del motor. }\end{array}$ & $\begin{array}{l}\text { Piezas móviles para el borde anterior de los } \\
\text { estabilizadores, sistemas conexos (servomo- } \\
\text { tores, descongeladores), paneles y piezas } \\
\text { secundarias de la parte central del fuselaje } \\
\text { (incluidos los accesorios de las alas). }\end{array}$ \\
\hline
\end{tabular}

Fuente: elaborado por el autor a partir de información proporcionada por EMBRAER y otras fuentes.

han apoyado activamente este proceso y contribuido respectivamente con $22 \%$ y $100 \%$ de los costos de desarrollo de la línea ERJ-145/135 y del caza liviano de ataque AL-X a reacción. Además, la EMBRAER ha sido la principal beneficiaria del Programa de Desarrollo Tecnológico Industrial (PDTI) del mismo ministerio, que brinda recursos nuevos y exoneraciones de impuestos a las empresas innovadoras. ${ }^{38} \mathrm{El}$ monto total asignado a la EMBRAER en calidad de subsidios entre 1993 y 2000 fue de 142 millones de reales. ${ }^{39}$ El FinAmex (actualmente BNDES-exim) le permite al BNDES financiar hasta el $100 \%$ de las exportaciones de bienes de capital. ${ }^{40}$

\footnotetext{
${ }^{38}$ Véase detalles del funcionamiento del mecanismo de promoción en http://www.mct.gov.br/prog/empresa/pdti_pdta/Default.htm.

${ }^{39}$ Datos obtenidos de http://www.mct.gov.br/prog/empresa/pdti pdta/ pdti res 20 12.htm.
}

El Programa de Estímulo a las Exportaciones (PROEX), administrado por el Banco do Brasil, es un programa de nivelación de los intereses en virtud del cual se rebaja hasta en $3.5 \%$ los intereses devengados por los créditos que se otorgan a los compradores de aviones brasileños exportados. La idea es contrarrestar el denominado Custo Brasil, esto es, el mayor riesgo que entraña hacer negocios en el país debido a la existencia de una serie de factores estructurales.

En este ambiente ferozmente competitivo, la rivalidad entre la EMBRAER y Bombardier se ha agravado a tal punto que ha dejado de ser un conflicto entre empresas y se ha convertido en cuestión nacional. El cuadro 6 resume las diversas etapas de esta controversia,

\footnotetext{
40 A fines de marzo de 2000 , poco más de la mitad de los trabajos pendientes eran financiados por el programa BNDES-exim (EMBRAER, 2000a, p. 12 .
} 
CUADRO 6

Brasil y Canadá: Controversia ante la OMC sobre créditos a las exportaciones de aviones de transporte regional

\begin{tabular}{lll}
\hline Acontecimiento & Fecha & Descripción \\
\hline
\end{tabular}

Enero de 1998

Informe de los enviados especiales

Mayo de 1998

Solicitud de Canadá a la OMC

Julio de 1998

Solicitud de Brasil a la Organización Mundial de Comercio (OMC)

Designación de los miembros de los Paneles

Decisión del Organo de Solución de Diferencias

Decisión del Organo de Solución de Diferencias

Decisiones del Organo de Apelación Informe de situación de Brasil

Informe de situación de Canadá

Convenio bilateral

Fallo del panel para la ejecución de las sentencias

Decisión del Organo de Apelación

Decisión del Panel arbitral

Decisión provisional

Fallo provisional

Fallo del Organo de Solución de Diferencias
Junio de 2001

Julio de 1998

Octubre de 1998

Marzo de 1999

Abril de 1999

Agosto de 1999

Noviembre de 1999

Noviembre de 1999

Noviembre de 1999

Mayo de 2000

Julio de 2000

Agosto de 2000

Octubre de 2001

Febrero de 2002
El Presidente Cardoso (Brasil) y el Primer Ministro Chrétien (Canadá) designan enviados especiales para que encuentren maneras de resolver la controversia.

Recomendación de que ambos gobiernos negocien un convenio bilateral en el plazo de dos meses.

Examinar si el componente de "nivelación de intereses" de PROEX constituye subsidio con arreglo al Artículo 1 del Agreement on Subsidies and Countervailing Measures (ASCM), y si tales subsidios contravienen el Artículo 3 de ese Convenio.

Examinar el financiamiento de las exportaciones proporcionado por la Export Development Corporation and Industry (EDC), de Canadá, para respaldar investigación y desarrollo.

La Unión Europea y los Estados Unidos se reservan el derecho a tercería en ambos procesos.

Brasil otorgó a EMBRAER un subsidio ilegal y debe dejarlo sin efecto en el plazo de 90 días.

Canadá debe poner fin de inmediato a algunos programas de subsidios que favorecen a Bombardier. No hay causa suficiente para seguir dando curso a las quejas de Brasil acerca del financiamiento otorgado por la EDC a los mercados secundarios.

Confirma las conclusiones iniciales en ambos casos.

Los pagos del PROEX para nivelación de los intereses sólo son admisibles en la medida en que el interés neto aplicable a una transacción con arreglo a ese programa se rebajen al nivel de la tasa de interés internacional de referencia correspondiente (y no menos, como ha sido el caso en el pasado).

El financiamiento otorgado por la Cuenta Canadá para la exportación de aviones de transporte regional se dejó sin efecto de inmediato, y se puso fin a todos los compromisos existentes de entrega de fondos del Technology Partnerships Canada (TPC) a esa industria.

Ambas partes solicitaron que se convocara nuevamente a los paneles originales del Organo de Solución de Diferencias, a fin de que decidan si ambas partes han cumplido sus decisiones.

No se pueden continuar las ventas basadas en el PROEX (unos 900 aviones fabricados por la EMBRAER).

Confirma la decisión anterior.

Hasta que se suscriba un convenio bilateral, Canadá tiene derecho a gravar mercancías provenientes de Brasil hasta por 230 millones de dólares al año.

El PROEX modificado no contraviene necesariamente la normativa de comercio internacional. Para que no haya provecho abusivo del programa por parte de Brasil, el financiamiento debe pactarse a los tipos de interés de mercado más una prima por concepto de riesgo; el plazo de pago de los préstamos no podrá ser superior a 10 años; y los créditos otorgados por el PROEX deben cubrir como máximo un $85 \%$ de la compra de que se trate.

Canadá solicitó la continuación del financiamiento otorgado a tres distintos compradores de aviones a reacción de Bombardier (Air Wisconsin, Comair y Air Nostrum).

La garantía a los préstamos de la Export Development Corporation (EDC) a tres distintos compradores de aviones a reacción de Bombardier constituía un subsidio ilegal a las exportaciones y debería dejarse sin efecto en el plazo de 90 días. 
que en mayo de 2000 llevó al gobierno canadiense a pedir a la OMC que le autorizara a aplicar medidas compensatorias y bloquear 3.3 mil millones de dólares en mercancías y ventajas comerciales a lo largo de siete años - lo que constituye la mayor confrontación comercial que haya tenido Canadá y una de las más importantes que se hayan planteado en la historia de la OMC. Las autoridades brasileñas replicaron que la amenaza de sanciones 'podría dificultar e incluso impedir que Brasil buscara alternativas que impidieran un agravamiento irracional del conflicto, y podría dar lugar a contramedidas u otro tipo de medidas que podrían perjudicar la relación económica y comercial en otras materias'. A partir de entonces, nuevas decisiones adoptadas por la OMC han favorecido a Brasil, y Canadá ha informado que cumplirá lo dispuesto por la decisión de febrero de 2002. Las conclusiones de un estudio conexo

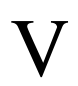

\section{Conclusiones}

Se ha dicho que la transformación de la EMBRAER es 'una leyenda que nos lleva de la zozobra al esplendor' (Exame, 1999) y que la empresa misma es un 'sobreviviente agresivo, osado y astuto' (The New York Times, 2000). Hay riesgos, por cierto: entre ellos destacan la marcada dependencia de un número reducido de clientes claves - Continental Express, American Eagle y Crossair-, el monto de la deuda y las consecuencias de competir frontalmente con Airbus y Boeing en el segmento inferior del mercado de aviones a reacción. Pero, al parecer, la situación de la empresa le permite resistir la mayoría de las perturbaciones.

La situación tanto interna como internacional obligó a Brasil a modificar la manera de abordar el desarrollo. Una de esas modificaciones fue la privatización, cambio fundamental para la EMBRAER, pues el traspaso de propiedad la forzó a adaptarse a un escenario nuevo en que la tecnología variaba rápidamente, los ciclos productivos eran más cortos y menguaba la capacidad financiera del Estado. El éxito con que dicha empresa opera en una industria tradicionalmente dominada por productores norteamericanos y europeos revela que las empresas de los países en desarrollo son capaces de adaptarse a circunstancias cambiantes y a condiciones adversas. Para hacerlo con éxito, tienen que canalizar los recursos hacia nuevas
(Goldstein y McGuire, 2002) destacan que las ideas de las autoridades políticas acerca de los objetivos de la política comercial han influido fuertemente en la prolongación de esta controversia. En el caso de Brasil, el liderazgo que ejerce en el mundo en desarrollo se convierte en un motivo para que cierren filas el gobierno y las empresas. En el caso de Canadá, su fe en un sistema de comercio basado en las reglas le ha llevado a oponerse con energía a cualquier tipo de violación, mientras que su incertidumbre en cuanto a su competitividad se ha traducido en una serie de programas gubernamentales de apoyo a las empresas de sectores modernos, como el aeroespacial. Esta investigación también concluye que, en la práctica, la OMC ha dificultado la solución del problema al hacer que tanto para las empresas como para los países perder resulte demasiado oneroso.

actividades generadoras de valor mediante el desarrollo de productos, la formación de alianzas y la toma de decisiones estratégicas. La capacidad de gestión debe concebirse como la habilidad para establecer una red de vinculaciones con diversos grupos externos a fin de aprovechar al máximo los recursos de la empresa.

Cabe preguntarse cuánta continuidad hay en esta historia. La empresa repuntó después de la privatización porque los nuevos administradores introdujeron formas nuevas de organizar el diseño, la producción, el financiamiento y la comercialización y redujeron severamente el tiempo que el producto tardaba en llegar al mercado. Sin embargo, no hay que olvidar que el proyecto de fabricación del ERJ-145, del cual tanto depende el destino de la EMBRAER, nació antes de que cambiara de dueño. Otra constante en la evolución de EMBRAER es ciertamente su capacidad de valerse de alianzas para atraer hacia la empresa recursos nuevos provenientes de fuentes externas. Puesto que la empresa compite con entidades más grandes de países que disponen de una mayor dotación de recursos y más infraestructura institucional, la EMBRAER obligadamente debió basar su desarrollo en la identificación de un núcleo reducido de capacidades y su aprovechamiento a lo largo del tiempo. Sin embargo, llama la atención lo que varió en términos relativos su 
poder de negociación. En los años setenta, la EMBRAER se vio favorecida por el hecho de que, para ingresar al mercado de Brasil y de otros países del sur, hubo empresas extranjeras de montaje que estuvieron dispuestas a otorgar licencias para utilizar su tecnología a una industria que todavía era de poca monta. Dos decenios después, sus socios se hallan entre los proveedores de nivel II de piezas para la industria aeroespacial —algunos de ellos de los países de la OCDE-, deseosos de aprovechar la credibilidad de que goza la EMBRAER para ingresar en el mercado mundial. ${ }^{41} \mathrm{La}$ reciente asociación con empresas francesas también es innovadora, ya que permite a la EMBRAER integrar su propia capacidad productiva a las técnicas de comercialización de sus aliados en Asia y en el mercado de la defensa.

Todas las empresas grandes y prósperas del mundo están convencidas de que deben igualar a sus competidores en tecnología, productividad y espíritu empresarial. Cabe preguntarse si la EMBRAER, que es una de las pocas empresas de América Latina que compite a nivel mundial, es diferente. ${ }^{42}$ A primera vista no parece que lo fuera, porque es tan agresiva, tecnológicamente avanzada y orientada al exterior como cualquier empresa en proceso de desarrollo acelerado. En otras palabras, lo que se intuye es que, sea cual fuere el país a que pertenezcan, las únicas empresas que pueden prosperar son aquellas capaces de obtener una buena rentabilidad de la inversión en instalaciones fabriles, sistemas de comercialización y métodos de gestión modernos (Dosi, Nelson y Winter, 2000). Al mismo tiempo, 'los países y las organizaciones abordan el desarrollo y la globalización de distintas maneras' (Guillén, 2000, p. 16). Por lo general, las razones son virtuosas: vale la pena ser diferentes. En consecuencia, lo sorprendente es que la EMBRAER deba internalizar algunas funciones que casi en todas partes distan mucho de ser responsabilidad directa de una empresa. La EMBRAER sólo llegó a contar con una fuerza de trabajo totalmente alfabetizada hacia la Navidad de 1997, oportunidad en que casi 150 empleados obtuvieron su licencia secundaria tras participar en un programa es-

\footnotetext{
41 SONACA y ENAER sólo se establecieron en 1978 y 1981, respectivamente; la colaboración de SONACA con la EMBRAER en 2001 representó un $55 \%$ de su volumen de negocios. GAMESA creó su división de aeronáutica para participar en el proyecto de fabricación de los ERJ-145, que representa un $90 \%$ de su cifra de negocios.

${ }^{42}$ Los argumentos que se plantean en este párrafo tendrían más fuerza si, como mínimo, yo hubiese comparado a la EMBRAER con Bombardier, a fin de examinar de qué manera crearon y explotaron sus capacidades organizacionales.
}

pecial de la empresa (Bernardes, 2000a). En 2002, el Instituto EMBRAER de Educación e Investigación inauguró una escuela secundaria especial en São José dos Campos (Colegio Eng. Juarez Wanderley).

Otra singularidad de la EMBRAER es que pese a tener una importante participación en el mercado y a que sus resultados financieros son satisfactorios, sigue siendo mucho más pequeña que competidores como Boeing, Airbus y Bombardier (cuya división aeroespacial forma parte de un grupo mucho más grande). El tamaño es importante en industrias como la aeronáutica, en que la habilidad para capear tormentas cíclicas asegura que el personal especializado y la maquinaria costosa sean utilizados incluso en las fases descendentes del ciclo, situación a la cual la EMBRAER ya debió hacer frente a comienzos del decenio de 1990. En consecuencia, el hecho de que la EMBRAER prospere como empresa independiente y no como división de uno de los grupos diversificados de empresas que tradicionalmente han dominado el panorama empresarial en los mercados emergentes, revela que es capaz de sacar el máximo de provecho de sus recursos.

Finalmente, hay otra enseñanza que la historia de la EMBRAER puede dejar a las autoridades económicas de los países en vías de industrialización. En sus primeros pasos como actor incipiente en el mercado mundial de aeronaves de transporte diario de pasajeros, la EMBRAER recibió apoyo del gobierno a través de diversos mecanismos, como la promoción tecnológica focalizada, el financiamiento y la capacitación. Debido a la importancia de objetivos no económicos, como el orgullo y prestigio nacionales, las decisiones adoptadas por el Ministerio de Aeronáutica en materia de adquisiciones se adaptaron a la meta de largo plazo de afianzar la acumulación de tecnología autóctona. Cabe subrayar que en el decenio de 1990 los órganos gubernamentales que apoyaban a la empresa variaron notablemente. Mientras que durante muchos años casi todos los defensores de la empresa pertenecían a la Fuerza Aérea y a círculos técnicos, últimamente es posible encontrarlos en el BNDES e incluso en Itamaraty (el Ministerio de Relaciones Exteriores de Brasil). ${ }^{43} \mathrm{Si}$ bien es cierto que en el pasado a los brasileños les enorgullecía contar con una empresa nacional capaz de fabricar aviones, ahora les complace aun más que la

\footnotetext{
${ }^{43}$ El gobierno de Brasil ha propuesto poner en marcha una iniciativa del Mercosur para incrementar el tamaño del mercado de transporte aéreo regional y está estudiando la posibilidad de derogar gravámenes nacionales que actualmente favorecen a las aeronaves importadas (O Estado de S. Paulo, 2000).
} 
EMBRAER pueda ganar dinero. Por su parte, los militares admiran mucho más los éxitos del programa aeroespacial brasileño y miran en menos las exportaciones de la EMBRAER, haciendo hincapié en que sus productos tienen un elevado contenido de importaciones. ${ }^{44}$

Así, la embraer ha sido capaz de influir en la estructura del mercado mundial de aviones regionales a reacción, en especial mediante subsidios a las exportaciones, pero, de manera más general, gracias al apoyo que obtuvo del gobierno del Brasil. Como la política para la industria aeronáutica no apuntaba a gravar los productos importados con aranceles altos ni a imponer exigencias en materia de contenido local, la adaptación a las normas de la OMC no ha sido difícil. Por supuesto, la principal excepción son los subsidios a las exportaciones, aunque hay que destacar que el PROEX es un mecanismo de base amplia que no se orienta a ningún sector determinado. Aunque de haber habido mayor empeño por reducir las fallas de la política en materia de oferta no habría sido tan necesario establecer un programa de nivelación de los tipos de interés (Bora, Lloyd y Pangestu, 2000), lo más probable es que la EMBRAER no hubiese logrado sobrevivir en el mundo real a la espera de que Brasil alcanzara ese grado de desarrollo. Naturalmente, para cuantificar los resultados del PROEx hay que realizar un análisis más riguroso, por mucho que a primera vista todo indica que ha permitido que la EMBRAER imponga los precios en su mercado. Lo mismo puede decirse respecto del financiamiento proporcionado por el BNDES, que prestó una ayuda decisiva a la gerencia en la etapa de recuperación, antes de que EMBRAER accediera a la Bolsa de Comercio de Nueva York. Aunque carezca de valor normativo, otra enseñanza más inmediata del conflicto Brasil-Canadá ante la OMC es que posiblemente sea más fácil sorprender aplicando políticas comerciales estratégicas a los países que no pertenecen a la OCDE - a lo mejor porque no se sientan a la misma mesa en que se llevan a cabo las negociaciones para regular los subsidios a las exportaciones.
Araujo, C. y J. L. Da Cruz (2000): A view of the practice of integrated product development at EMBRAER, trabajo presentado al $5^{\circ}$ Seminario internacional de alta tecnología, Piracicaba, 5 de octubre, inédito.

Aviation Week and Space Technology (2001): 2000 Laurels Awards, 7 de mayo.

Baldwin, R. y H. Flam (1989): Strategic trade policies en the market for 30-40 seat commuter aircraft, Weltwirtschaftliches Archiv, $\mathrm{N}^{\circ} 125$, Tübingen, J.C.B. Mohr.

Bernardes, R. (2000a): EMBRAER. Elos entre Estado e mercado, Hucitec.

(2000b): O arranjo produtivo da EMBRAER na região de São José dos Campos, trabajo presentado al Seminario internacional Arranjos e Sistemas Produtivos Locais e as Novas Políticas de Desenvolvimento Industrial e Tecnológico (Rio de Janeiro, 4 al 5 de septiembre), inédito.

Bora, B., P. Lloyd, y M. Pangestu (2000): Industrial policy and the WTO, World Economy, vol. 23, N 4, Oxford, Reino Unido, Blackwell Publishers.

Broinizi Pereira, M. (1991): Tiro pela culatra, en Fundação Perseu Abramo, Teoria \& debate, $\mathrm{N}^{\circ} 14$.

Busch, M. (1999): Trade Warriors. States, Firms, and StrategicTrade Policy in High-Technology Competition, Cambridge, Massachusetts, Cambridge University Press.

Business Week (1996): EMBRAER's little jet could circle the globe, Estados Unidos, McGraw Hill Companies, Inc., 28 de octubre.

Campolina Diniz, C. y M. Razavi (1999): São José dos Campos and Campinas, en A. Markusen, Y. S. Lee y S. DiGiovanna (eds.),

\footnotetext{
44 Entrevista con el Director General del Departamento de Investigación y Desarrollo de la Fuerza Aérea (en Tecnologia \& Defesa, 1999).
}

Second Ties Cities, Minneapolis, University of Minnesota Press.

Chandler, A., F. Amatori y T. Hikino (1997): Big Business and the Wealth of Nations, Cambridge, Reino Unido, Cambridge University Press.

Dagnino, R. (1993): Competitividade da indústria aeronáutica, Brasilia, Ministerio de Ciencia y Tecnología / Fondo para el Financiamiento de Estudios y Proyectos (FINEP)/ Programa de Apoyo al Desarrollo Científico y Tecnológico (PADCT).

Dahlman, C. y C. Frischtak (1993): National systems supporting technical advance in industry: The Brazilian experience, en R. Nelson (ed.), National Innovation Systems, Oxford, Reino Unido, Oxford University Press.

Dosi, G., R. Nelson y S. Winter (2000): Introduction: The nature and dynamics of organizational capabilities, en G. Dosi, R. Nelson, y S. Winter (eds.), The Nature and Dynamics of Organizational Capabilities, Oxford, Reino Unido, Oxford University Press.

EMBRAER (Empresa Brasileña de Aeronáutica) (2000a): Prospectus, São João dos Campos, Brasil.

(2000b): Balanço Social, São João dos Campos, Brasil.

Evans, P. (1979): Dependent Development, Princeton, New Jersey, Princeton University Press.

Exame (1999): Decolou!, 30 de junio.

Financial Times (2000): Regional jet market gains altitude over bigger rivals, Londres, 17 de abril.

Folha de S. Paulo (2000): EMBRAER quer exportar US\$9bi até 2005, São Paulo, 5 de noviembre (www.folha.vol.com.br/folha).

Frischtak, C. (1992): Learning, Technical Progress and Competitiveness in the Commuter Aircraft Industry: An Analysis of EMBRAER, Industry series paper, $\mathrm{N}^{\circ} 58$, Washington, D.C., Banco Mundial, Departamento de Industria y Energía.

Fujimoto, T. (2000): Evolution of manufacturing capabilities and ex post dynamic capabilities: A case of Toyota's final 
assembly operations, en G. Dosi, R. Nelson y S. Winter (eds.), The Nature and Dynamics of Organizational Capabilities, Oxford, Reino Unido, Oxford University Press.

Galambos, S. (2000): Regional/business aircraft and parts: Switzerland, Industry Sector Analysis Series, U.S. Commercial Service and U.S. Department of State.

Gazeta Mercantil (2000): Fornecedores da EMBRAER abrem fábricas no país, 8 de noviembre (www. investnews.net).

Ghemawat, P., G. Herrero y L. Monteiro (2000): EMBRAER: The global leader in regional jets, Harvard Business School Case Studies, Working paper, $\mathrm{N}^{\circ}$ N9-701-006, Boston, Massachusetts, Harvard Business School Press.

Goldberg, P. y G. Maggi (1999): Protection for sale: An empirical investigation, The American Economic Review, vol. 89, $\mathrm{N}^{\circ} 6$, Nashville, Tennessee, American Economic Association.

Goldstein, A. y S. McGuire (2002): The political economy of strategic trade policy and the Brazil-Canada export subsidies saga, París, Organización de Cooperación y Desarrollo Económicos (OCDE), Centro de Desarrollo, inédito.

Guillén, M. (2000): Diversity in Globalization. Organizational Change in Argentina, South Korea and Spain, Occasional papers, $\mathrm{N}^{\circ} 6$, Princeton, New Jersey, Princeton University, School of Social Science.

$O$ Estado de S. Paulo (2000): Maciel sugere que embraer cresça no Mercosul, São Paulo, 22 de septiembre.

Ramamurti, R. (1987): State-Owned Enterprises in High Technology Industries, Nueva York, Praeger.
Sbragia, R. y J. Terra (1993): EMBRAER: trajetória de uma empresa de alta tecnologia brasileira, Cadernos de gestão tecnológica, $\mathrm{N}^{\circ}$ 8, São Paulo, Núcleo de Política e Gestão de Ciência y Technologia (NPGCT)/ Universidad de São Paulo.

Sikkink, K. (1991): Ideas and Institutions: Developmentalism in Brazil and Argentina, Ithaca, Nueva York, Cornell University Press.

Silva, O. (1998): A decolagem de um sonho. A história da criação da embraer, São Paulo, Lemos.

Solingen, E. (1993): Macropolitical consensus and lateral autonomy in industrial policy: The nuclear sector in Brazil and Argentina, International Organization, vol. 47, $\mathrm{N}^{\circ} 2$, Cambridge, Massachusetts, The MIT Press.

(1998): Growth and decline of the military-industrial complex: The cases of Argentina and Brazil, International Politics, vol. $35, \mathrm{~N}^{\circ} 1$.

Tecnologia \& Defesa (1999): Dificil criar: fácil de desaparecer, $\mathrm{N}^{\circ} 82$, São Paulo.

The Daily Telegraph (1999): BA eyes 20pc stake in EMBRAER, Reino Unido, 14 de agosto (www.dailytelegraph.co.uk).

The New York Times (2000): Brazil's hot commodity? Not coffee or soccer, Nueva York, 31 de diciembre.

Thomke, S. y T. Fujimoto (2000): The effect of 'front-loading' problem-solving on product development performance, Journal of Product Innovation Management, vol. 17, $\mathrm{N}^{\circ} 1$.

Trebat, T. J. (1983): Brazil's State-Owned Enterprises: A Case Study of the State as Entrepreneur, Cambridge, Reino Unido, Cambridge Universiy Press.

Veja (2000): De cavalo a burro, 12 de enero. 